Article

\title{
The Structure-Activity Relationship between Marine Algae Polysaccharides and Anti-Complement Activity
}

\author{
Weihua Jin ${ }^{1,3}$, Wenjing Zhang ${ }^{1,2}$, Hongze Liang ${ }^{4}$ and Quanbin Zhang ${ }^{1,3, *}$ \\ Received: 30 July 2015; Accepted: 13 December 2015; Published: 25 December 2015 \\ Academic Editor: Gerald Murphy \\ 1 Key Laboratory of Experimental Marine Biology, Institute of Oceanology, Chinese Academy of Sciences, \\ Qingdao 266071, China; jinweihua@qdio.ac.cn (W.J.); wenjingwing@126.com (W.Z.) \\ 2 College of Earth Science, University of Chinese Academy of Sciences, Beijing 100049, China \\ 3 Laboratory for Marine Biology and Biotechnology, Qingdao National Laboratory for Marine Science and \\ Technology, Qingdao 266071, China \\ 4 The School of Materials Sciences and Chemical Engineering, Ningbo University, Ningbo 315211, China; \\ lianghongze@nbu.edu.cn \\ * Correspondence: qbzhang@qdio.ac.cn; Tel.: +86-532-8289-8703; Fax: +86-532-8289-8703
}

\begin{abstract}
In this study, 33 different polysaccharides were prepared to investigate the structure-activity relationships between the polysaccharides, mainly from marine algae, and anti-complement activity in the classical pathway. Factors considered included extraction methods, fractionations, molecular weight, molar ratio of galactose to fucose, sulfate, uronic acid (UA) content, linkage, branching, and the type of monosaccharide. It was shown that the larger the molecular weights, the better the activities. The molar ratio of galactose (Gal) to fucose (Fuc) was a positive factor at a concentration lower than $10 \mu \mathrm{g} / \mathrm{mL}$, while it had no effect at a concentration more than $10 \mu \mathrm{g} / \mathrm{mL}$. In addition, sulfate was necessary; however, the sulfate content, the sulfate pattern, linkage and branching had no effect at a concentration of more than $10 \mu \mathrm{g} / \mathrm{mL}$. Moreover, the type of monosaccharide had no effect. Laminaran and UA fractions had no activity; however, they could reduce the activity by decreasing the effective concentration of the active composition when they were mixed with the active compositions. The effect of the extraction methods could not be determined. Finally, it was observed that sulfated galactofucan showed good anti-complement activity after separation.
\end{abstract}

Keywords: marine algae polysaccharides; anti-complement activity; structure-activity relationship

\section{Introduction}

The complement system is an important part of the innate immune system that is designed to eliminate "harmful" substances from the body. This elimination is accomplished in five different ways according to a previous study [1]. Inappropriate activation of the complementary target self-tissues causes pathology in a large number of inflammatory, ischaemic, and other diseases [2].

There is a large body of research on naturally-occurring complement inhibitors isolated from animal, plant, and microbial products, such as polysaccharides [3-6], phenolic acid [7], proteins [8], flavonoids, and steroides [9,10]. Many synthetic molecules, including dextran sulfate [11,12], nafamastat mesilate (FUT-175) [2] and compstatin [1] have been shown to inhibit activation of the complement system. In addition, many studies have also reported [13-15] that a sulfated polysaccharide from brown algae, has been found to inhibit complement activation. Structure and activity studies showed that sulfated polysaccharides are potential candidates for screening as inhibitors of the complement system. Marine algae contain numerous different polysaccharides, 
which are non-toxic. In addition, they also contain polyanions and are majorly substituted by sulfate, which differs from the polysaccharides from land-plants.

Currently, marine algae mainly contain brown algae, red algae, and green algae. Polysaccharides from brown algae [16-21] contain three major types of polysaccharides, namely, fucoidan, laminaran, and alginate. Fucoidan includes sulfated heteropolysaccharides and sulfated galactofucan or fucan. The former contains sulfated glucuronomannan, glucuronan, galactan, etc. Sulfated glucuronomannan has a backbone of alternating 2-linked mannopyranose residue and 4-linked glucuronic acid, sulfated at $\mathrm{C} 6$ of the mannnopyranose residue. The linkage of glucuronan is 3-linked. Galactan has a backbone of 6-linked galactopyranose residue branched at $\mathrm{C} 4$ with galactose, while the latter one is made up of 3-linked fucopyranose residues or alternating 3-linked and 4-linked fucopyranose residues. The major differences between sulfated galactofucan or fucan are the positions of the sulfate and branching units, including the fucose residues, galactose residues, glucuronic acid residues, and so on. Laminaran is a glucan, consisting mainly of 3-linked glucopyranose residue, branched with 6-linked glucopyranose. Alginate contains polymannuronic acid, polyguluronic acid and a mixture of polymannuronic acid and polyguluronic acid. Polysaccharides from red algae [18,21-24] contain three types of polysaccharides, namely, carrageenan polysaccharides, agar polysaccharides, and an agar-carrageenan intermediate polysaccharide. They have a backbone of alternating 3 -linked $\beta$-D-galactopyranose and 4-linked $\alpha$-galactopyranose residues; however, carrageenan polysaccharides have 4 -linked $\alpha$-D-galactopyranose residues while agar polysaccharides have 4 -linked $\alpha$-L-galactopyranose residues. In addition, all the $\alpha$-galactopyranose residues might exist in the form of 3,6-substituted by ester sulfate, methyl groups, pyruvic acid acetal, etc. Water-soluble polysaccharides from green algae $[18,23,25]$ can be divided into two types of polysaccharides. Type 1 is classified as xylogalactoarabian, which mainly consists of xylose, galactose, and arabinose. It contains a backbone of 4-linked or 5-linked arabinopyranose residues sulfated at C3, 3-linked or 6-linked galactopyranose residues sulfated at $\mathrm{C} 4$ or $\mathrm{C} 6$, and 4-linked xylopyranose residues. Type 2 is named as glucuronoxylorhamnan, which mainly contains glucuronic acid, xylose, and rhamnose, according to the type of major monosaccharides. It consists of 4-linked rhamnopyranose sulfated at C3 or C2, a 4-linked glucuronic acid residue and a 4-linked xylopyranose residue.

To clarify the structure-activity relationship between marine algae polysaccharides and their anti-complement activity, several types of polysaccharides from brown algae, red algae, and green algae were prepared. In addition, many factors including the extraction methods, fractionations, molecular weight, the molar ratio of galactose to fucose, sulfate, UA content, linkage, branching, and the type of monosaccharides were taken into account.

\section{Results and Discussion}

\subsection{The Effect of the Extraction Methods on the Anti-Complement Activity}

Table 1 displays the chemical composition of two fucoidans, which were derived from Saccharina japonica by hot water (SJW) and diluted hydrochloric acid (SJS). It was shown that the contents of fucose (Fuc) and sulfate contained in SJS were higher than in SJW, while the content of UA contained in SJS was lower. In addition, the molecular weight of SJS was also lower because of degradation during the process of extraction by dilute hydrochloric acid. Moreover, it also indicated that SJW contained a greater number of other monosaccharides, suggesting that the structure of SJW was more complex than that of SJS. In terms of the chemical compositions of HFW (HFW was obtained from brown algae Hizikia fusiforme by hot water) and the HFS (HFS was obtained from brown algae Hizikia fusiforme by dilute hydrochloric acid), as shown in Table 1, Fuc, UA and the molecular weight of the HFW were higher than in HFS, while the sulfate content of HFW was lower. The molar ratios of monosaccharides indicated that both HFS and HFW contained substantial amounts of laminaran, which was also reported in a previous study [26]. To summarize, different extraction methods might influence the chemical compositions of the polysaccharides. 
Table 1. Chemical composition (\%, dry weight) of the polysaccharides studied.

\begin{tabular}{|c|c|c|c|c|c|c|c|c|c|c|c|c|}
\hline \multirow{2}{*}{ Sample } & \multirow{2}{*}{$\begin{array}{c}\text { Yields } \\
(\%)\end{array}$} & \multirow{2}{*}{$\begin{array}{l}\text { Fuc } \\
(\%)\end{array}$} & \multirow{2}{*}{$\begin{array}{l}\text { UA } \\
(\%)\end{array}$} & \multirow{2}{*}{$\begin{array}{c}\mathrm{SO} 4 \\
(\%)\end{array}$} & \multicolumn{7}{|c|}{ Monosaccharides (Molar Ratio) } & \multirow{2}{*}{ Mw (kDa) } \\
\hline & & & & & Man & Rha & GlcA & Glc & Gal & Xyl & Fuc & \\
\hline SJW & 1.21 & 22.38 & 10.32 & 30.60 & 0.10 & 0.03 & 0.15 & 0 & 0.63 & 0.07 & 1 & 152.4 \\
\hline SJW-1 & 10.67 & 15.84 & 33.36 & 15.05 & 0.55 & 0.10 & 0.96 & 0 & 1.22 & 0.37 & 1 & 77.8 \\
\hline SJW-2 & 19.99 & 17.01 & 17.35 & 28.33 & 0.12 & 0.03 & 0.18 & 0 & 1.08 & 0.10 & 1 & 88.4 \\
\hline SJW-3 & 37.89 & 28.96 & 0.45 & 35.10 & 0.02 & 0 & 0.02 & 0 & 0.27 & 0 & 1 & 162.7 \\
\hline SJS & 1.08 & 34.97 & 3.44 & 36.88 & 0.06 & 0.03 & 0.06 & 0.01 & 0.11 & 0.02 & 1 & 106.3 \\
\hline SJS-1 & 8.98 & 21.56 & 13.71 & 19.75 & 0.06 & 0.02 & 0.06 & 0.02 & 0.17 & 0.02 & 1 & 63.8 \\
\hline SJS-2 & 15.89 & 31.07 & 8.21 & 34.84 & 0.08 & 0.03 & 0.07 & 0.01 & 0.28 & 0.03 & 1 & 84.5 \\
\hline SJS-3 & 40.78 & 36.94 & 0 & 38.43 & 0.01 & 0 & 0.01 & 0 & 0.05 & 0 & 1 & 125.4 \\
\hline HFW & 2.43 & 28.04 & 5.43 & 28.46 & 0.11 & 0 & 0.08 & 0.62 & 0.27 & 0.09 & 1 & $118.3 / 3.9$ \\
\hline HFW-1 & 18.51 & - & - & - & - & - & - & 1 & - & - & - & 3.9 \\
\hline HFW-2 & 18.16 & 22.78 & 17.64 & 17.33 & 0.48 & 0.30 & 0.27 & 0.29 & 0.23 & 0.42 & 1 & $116.7 / 5.1$ \\
\hline HFW-3 & 28.01 & 32.85 & 0 & 31.62 & 0.05 & 0.01 & 0.04 & 0.01 & 0.29 & 0.04 & 1 & 114.2 \\
\hline HFS & 1.53 & 21.89 & 1.98 & 30.59 & 0.07 & 0.01 & 0.09 & 1.22 & 0.25 & 0.02 & 1 & $97.3 / 3.6$ \\
\hline HFS-1 & 16.24 & - & - & - & - & - & - & 1 & - & - & - & 3.4 \\
\hline HFS-2 & 10.66 & 25.79 & 20.84 & 25.66 & 0.27 & 0.02 & 0.20 & 0.03 & 0.22 & 0.04 & 1 & $42.0 / 4.3$ \\
\hline HFS-3 & 9.72 & 40.84 & 0.98 & 39.85 & 0 & 0 & 0 & 0 & 0.27 & 0.01 & 1 & 99.2 \\
\hline SJS-OS & 98.19 & 33.31 & 3.31 & 46.36 & 0.06 & 0.03 & 0.05 & 0.05 & 0.20 & 0.05 & 1 & 119.5 \\
\hline SJS-DS & 65.41 & 41.20 & 6.11 & 18.12 & 0.05 & 0.03 & 0.05 & 0.03 & 0.18 & 0.05 & 1 & $117.5 / 33.4$ \\
\hline SJS-DS-OS & 110.1 & 24.24 & 3.79 & 35.30 & 0.05 & 0.02 & 0.04 & 0.03 & 0.20 & 0.05 & 1 & 123.6 \\
\hline ANW & & 25.08 & 4.16 & 27.36 & 0.05 & 0.03 & 0.09 & 0.29 & 0.09 & 0.06 & 1 & 122.1 \\
\hline SC & 5.78 & 21.80 & 6.54 & 28.15 & 0.15 & 0.32 & 0.13 & 0.22 & 0.28 & 0.08 & 1 & 111.9 \\
\hline SJW-2-R & 38.99 & 18.56 & 9.86 & 29.63 & 0.10 & 0.03 & 0.06 & 0.10 & 1.05 & 0.07 & 1 & 79.6 \\
\hline SJW-2-HJ & 10.89 & 14.35 & 29.36 & 14.63 & 0.46 & 0.10 & 0.50 & 0 & 2.10 & 0.20 & 1 & 50.4 \\
\hline SJW-2-HW & 83.10 & 25.23 & 8.36 & 35.69 & 0.09 & 0.01 & 0.10 & 0 & 0.56 & 0.06 & 1 & 95.6 \\
\hline HFW-1-S & 109.1 & - & - & 41.38 & - & - & - & 1 & - & - & - & 13.7 \\
\hline ES & 23.19 & - & - & 26.33 & - & - & - & - & 1 & - & - & 147.9 \\
\hline GF & 13.30 & - & - & 20.42 & - & - & - & - & 1 & - & - & 137.2 \\
\hline GL & 30.28 & - & - & 2.74 & - & - & - & - & 1 & - & - & 146.0 \\
\hline PY & 10.71 & - & - & 2.72 & - & - & - & - & 1 & - & - & 140.7 \\
\hline $\mathrm{EP}$ & 19.53 & - & 26.77 & 18.09 & - & 1 & 0.37 & 0.13 & 0.06 & 0.31 & - & 189.3 \\
\hline UP & 18.75 & & 28.39 & 20.88 & - & 1 & 0.45 & 0.15 & 0.04 & 0.33 & - & 163.4 \\
\hline CR & 8.13 & - & - & 32.84 & - & 0.03 & - & - & 0.14 & 1 & - & 174.9 \\
\hline $\mathrm{CF}$ & 7.89 & - & - & 23.54 & - & 0.05 & - & 0.40 & 1 & 0.50 & - & 173.4 \\
\hline
\end{tabular}

Polysaccharides derived from Saccharina japonica by hot water (SJW) and dilute hydrochloric acid (SJS). SJW was fractionated by anion exchange chromatography into three components, namely SJW-1, SJW-2 and SJW-3. SJS was also fractionated by anion exchange chromatography into three components, namely SJS-1, SJS-2 and SJS-3. Polysaccharides derived from Hizikia fusiforme by hot water (HFW) and dilute hydrochloric acid (HFS). HFW was fractionated by anion exchange chromatography into three components, namely HFW-1, HFW-2 and HFW-3. HFS was also fractionated by anion exchange chromatography into three components, namely HFS-1, HFS-2 and HFS-3. Overly sulfated SJS (SJS-OS) and laminaran (HFW-1-S), desulfated SJS (SJS-DS) and desulfated SJS with sulfation (SJS-DS-OS). Polysaccharides derived from Ascophyllum nodosum (ANW), Acaudina molpadioides (SC), Eucheuma spinosum (ES), Grateloupia filicina (GF), Gracilaria lamaneiformis (GL), Porphyra yezoensis (PY), Enteromorpha prolifera (EP), Ulva pertusa (UP), Cladophera rupestris (CR) and Codium fragile (CF). SJW-2-R was obtained through UA reduction, and SJW-2-HJ and SJW-2-HW were fractionated using an activated carbon column.

Figure 1a displays the anti-complement activities of SJS, SJW, HFS and HFW in the classical pathway. Activities of SJS and HFW reached a plateau at a concentration of $10 \mu \mathrm{g} / \mathrm{mL}$, while SJW and HFS reached a plateau at a concentration of $50 \mu \mathrm{g} / \mathrm{mL}$. Specifically, the $\mathrm{IC}_{50}$ of SJS, SJW, HFS, and HFW were 4.51, 7.26, 24.65, and $5.51 \mu \mathrm{g} / \mathrm{mL}$, respectively. Thus, it was difficult to determine the effects of the extraction methods because the $\mathrm{IC}_{50}$ of SJS was lower than that of SJW, while the $\mathrm{IC}_{50}$ of HFW was lower than that of HFS. 

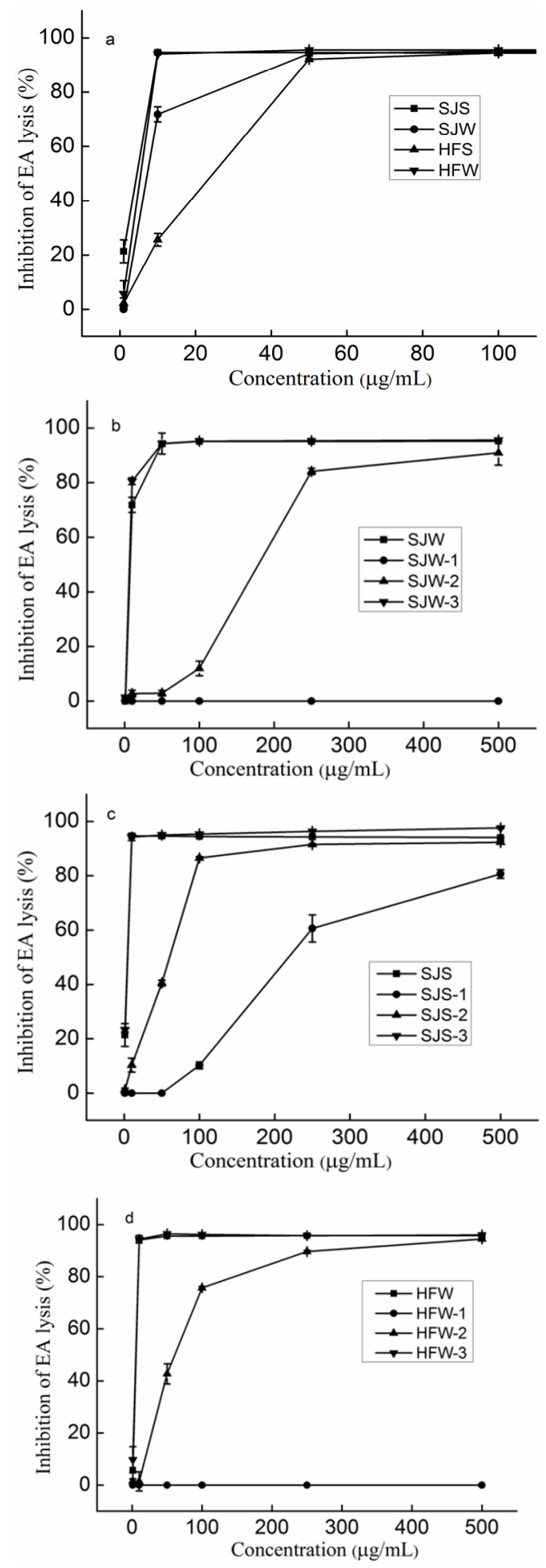

Figure 1. Cont. 


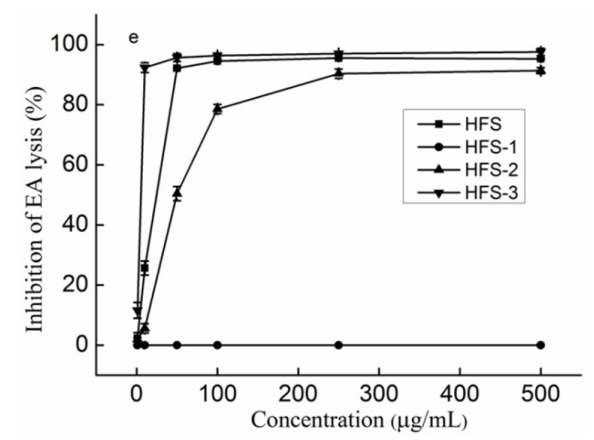

Figure 1. Inhibition of the classical pathway-mediated haemolysis of EA in 1:10-diluted NHS in the presence of increasing amounts of the polysaccharides (a) SJS, SJW, HFS, and HFW; (b) SJW and its fractions; (c) SJS and its fractions; (d) HFW and its fractions; (e) HFS and its fractions. The results are expressed as percent inhibition of haemolysis. Data are the means from three determinations \pm S.E.M. Polysaccharides derived from Saccharina japonica by hot water (SJW) and dilute hydrochloric acid (SJS). SJW was fractionated by anion exchange chromatography into three components, namely SJW-1, SJW-2 and SJW-3. SJS was also fractionated by anion exchange chromatography into three components, namely SJS-1, SJS-2 and SJS-3. Polysaccharides derived from Hizikia fusiforme by hot water (HFW) and dilute hydrochloric acid (HFS). HFW was fractionated by anion exchange chromatography into three components, namely HFW-1, HFW-2 and HFW-3. HFS was also fractionated by anion exchange chromatography into three components, namely HFS-1, HFS-2 and HFS-3.

\subsection{The Effect of the Fractionations on the Anti-Complement Activity}

SJW was fractionated by anion exchange chromatography into three components, namely, SJW-1, SJW-2, and SJW-3. Chemical analysis indicated that SJW-1 had the highest UA content and SJW-3 had the highest fucose content and sulfate content. In addition, SJW-3 had the highest molecular weight. Previous studies [27-29] reported that SJW-3 was mainly sulfated galactofucan, and SJW-1 and SJW-2 were sulfated heteropolysaccharides. Figure $1 \mathrm{~b}$ shows the anti-complement activities of SJW and its fractions. Compared to SJW and its fractions, it was found that the activity of SJW-3 was similar to the SHW activity, while SJW-2 had lower activity and SJW-1 had no activity. Specifically, the $\mathrm{IC}_{50}$ of SJW-3 $(3.11 \mu \mathrm{g} / \mathrm{mL})$ was lower than that of SJW $(7.26 \mu \mathrm{g} / \mathrm{mL})$. The chemical composition of SJS's fractions (SJS was fractionated by anion exchange chromatography into three components, namely, SJS-1, SJS-2, and SJS-3) were similar to the results of SJW's fractions, suggesting that SJS-3 was a sulfated galactofucan, while SJS-1 and SJS-2 were sulfated heteropolysaccharides. The activities of SJS and its fractions in Figure 1c confirmed the above results. HFW was also fractionated by anion exchange chromatography into three components, namely, HFW-1, HFW-2, and HFW-3. HFW-1 was composed of glucose, HFW-2 had plenty of the other monosaccharides and HFW-3 contained galactose and fucose. According to the chemical compositions in Table 1, it was proposed that HFW-1 was mainly laminaran, HFW-2 was mainly sulfated heteropolysaccharides, and HFW-3 was mainly sulfated galactofucan, which was in accordance with a previous study [26]. Figure $1 \mathrm{~d}$ also shows that HFW-3 had the best activity. HFS was fractionated by anion exchange chromatography into three components, namely, HFS-1, HFS-2, and HFS-3. Table 1 showed that HFS- 1 contained only glucose, HFS-2 had many other monosaccharides, while HFS-3 had galactose and fucose, suggesting that they were similar to the fractions of HFW. The activities of HFS and its fractions in Figure 1e also confirmed the above results. In conclusion, sulfated galactofucan contributed to the anti-complement activities.

\subsection{The Effect of Molecular Weight on the Anti-Complement Activity}

To study the relationship between the molecular weight and the anti-complement activity of sulfated galactofucan, six sulfated galactofucans (GF90, GF50, GF36, GF30, GF11, and GF8) (the 
molar ratio of galactose to fucose was approximately 0.08 ) with different molecular weights were determined. As described in the previous study [30], six sulfated galactofucans, namely GF90, GF50, GF36, GF30, GF11, and GF8, were prepared. The molecular weights of these were 90.1, 50.1, 36.0, 29.5, 11.3 , and $8.4 \mathrm{kDa}$, respectively. It is shown in Figure 2a that the activity increased as the molecular weights increased. To a certain extent, these results were consistent with previous studies [13,30]. Blondin et al. [13] reported that the anti-complement activities of sulfated fucan in the classical pathway increased with increasing molecular weight and reached a plateau at $40 \mathrm{kDa}$. In addition, the activities also showed dose dependence. GF90 and GF50 had the highest activities at a concentration of $50 \mu \mathrm{g} / \mathrm{mL}$, while GF8 did not reach the highest activities even at a concentration of $400 \mu \mathrm{g} / \mathrm{mL}$. Therefore, it was concluded that the molecular weight constituted an important positive factor on the activities.

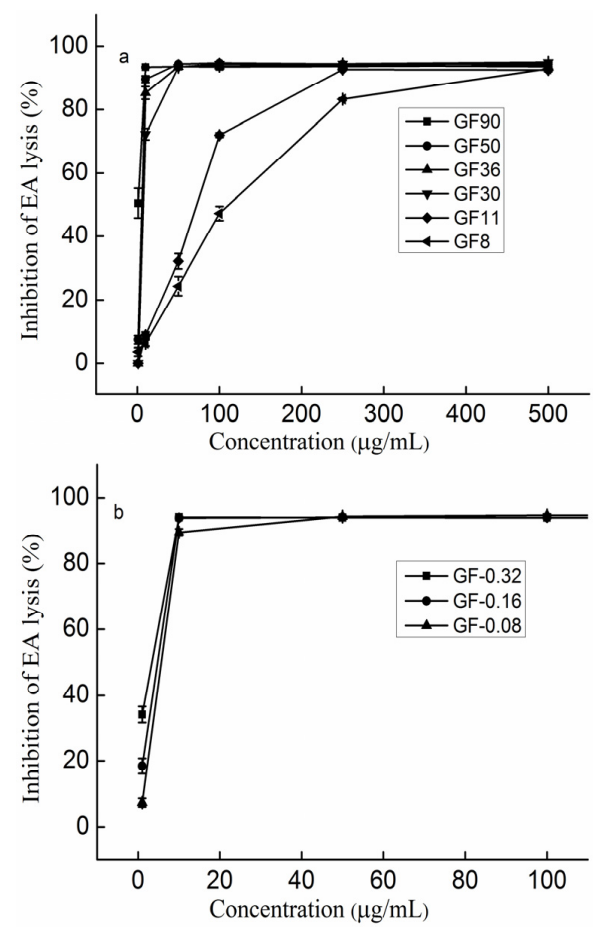

Figure 2. Inhibition of the classical pathway-mediated haemolysis of EA in 1:10-diluted NHS in the presence of increasing amounts of the polysaccharides (a) The different molecular weights of sulfated galactofucan; (b) Sulfated galactofucan with different molar ratios of galactose to fucose. The results are expressed as percent inhibition of haemolysis. Data are the means from three determinations \pm S.E.M. Six sulfated galactofucans weighed 90kDa (GF90), 50kDa (GF50), 36kDa (GF36), 30kDa (GF30), $11 \mathrm{kDa}(\mathrm{GF} 11)$, and $8 \mathrm{kDa}$ (GF8). Three sulfated galactofucans had a molar ratio of galactose to fucose of 0.32 (GF-0.32), 0.16 (GF-0.16) and 0.08 (GF-0.08).

\subsection{The Effect of the Molar Ratio of Galactose to Fucose on the Anti-Complement Activity}

The chemical compositions of GF-0.32, GF-0.16 and GF-0.08 (also sulfated galactofucans) were reported in a previous study [30]. It was shown that GF-0.32, GF-0.16, and GF-0.08 had similar chemical compositions, except for the molar ratio of galactose to fucose. GF- 0.32 weighed $49.3 \mathrm{kDa}$ with a molar ratio of galactose to fucose of 0.32 ; GF- 0.16 weighed $49.2 \mathrm{kDa}$ with 0.16 , and GF-0.08 weighed $50.1 \mathrm{kDa}$ with 0.08 . Figure $2 \mathrm{~b}$ shows that all samples reached the highest activities at a concentration of $10 \mu \mathrm{g} / \mathrm{mL}$. There was little difference in the activities of GF-0.32, GF-0.16, and GF- 0.08 at a concentration of $1 \mu \mathrm{g} / \mathrm{mL}$, indicating that the molar ratio of galactose to fucose contributed to the anti-complement activity. In conclusion, the anti-complement activity was attributed to the molar ratio of galactose to fucose. 


\subsection{The Effect of Sulfate on the Anti-Complement Activity}

Previous studies [31-33] have demonstrated the importance of sulfate in the biological activities of polysaccharides. Thus, overly sulfated polysaccharide (SJS-OS), desulfated polysaccharide (SJS-DS) and desulfated polysaccharide with sulfation (SJS-DS-OS) were prepared. Chemical compositions, shown in Table 1, indicated that SJS-OS's sulfate increased from $36.88 \%$ to $46.36 \%$, SJS-DS's sulfate decreased from $36.88 \%$ to $18.12 \%$, and SJS-DS-OS's sulfate increased from $18.12 \%$ to $35.30 \%$. Figure $3 \mathrm{a}$ indicates that SJS, SJS-OS and SJS-DS-OS reached the highest activities at a concentration of $10 \mu \mathrm{g} / \mathrm{mL}$, while SJS-DS had no activity. In addition, SJS-OS had the highest activity at a concentration of $1 \mu \mathrm{g} / \mathrm{mL}$, SJS-DS-OS second, SJS third and SJS-DS had no activity. It was suggested that a certain sulfate content was necessary for the activities. In addition, the activities did not increase along with increasing sulfate content at a concentration of more than $10 \mu \mathrm{g} / \mathrm{mL}$.

In addition to the effect of the sulfate content on the activities, the effect of the sulfate pattern was also elucidated by comparison of a pair of sulfated galactofucans (SJS-3 and HFS-3), which were fractionated from SJS and HFS by anion exchange chromatography. According to previous studies [26,28,34,35], SJS-3 is mainly sulfated at C-4 of Fuc, while HFS-3 is mainly sulfated at C-2. The molecular weights of SJS-3 and HFS-3 were $125.4 \mathrm{kDa}$ and $99.2 \mathrm{kDa}$, respectively, as shown in Table 1, suggesting that the effects of the molecular weight on the activity could be ignored. Moreover, the sulfate content of SJS-3 (38.43\%) was similar to HFS-3's (39.85\%). Figure 3b shows the activities of SJS-3 and HFS-3, which were similar to each other at a concentration of more than $10 \mu \mathrm{g} / \mathrm{mL}$.

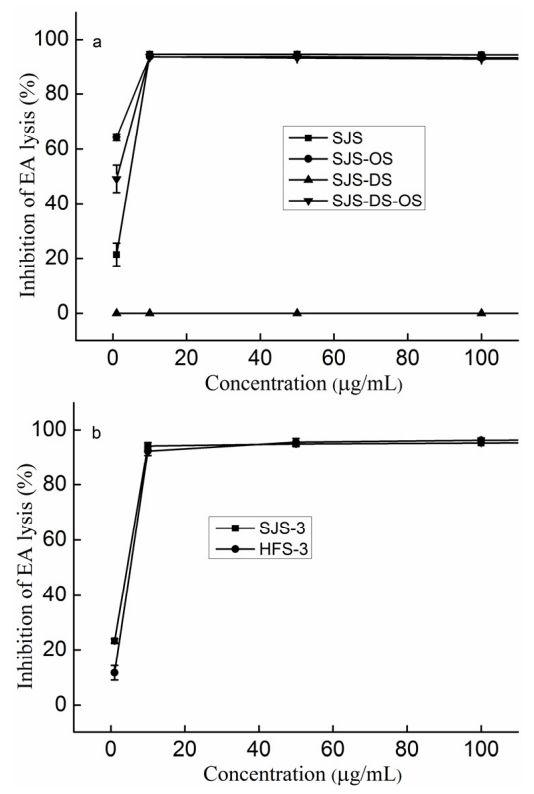

Figure 3. Inhibition of the classical pathway-mediated haemolysis of EA in 1:10-diluted NHS in the presence of increasing amounts of the polysaccharides (a) Polysaccharides with different sulfate contents; (b) Polysaccharides with different sulfate patterns. The results are expressed as percent inhibition of haemolysis. Data are the means from three determinations \pm S.E.M. Overly sulfated SJS (SJS-OS), desulfated SJS (SJS-DS) and desulfated SJS with sulfation (SJS-DS-OS).

Finally, it was concluded that the sulfate content was necessary for the activities; however, the effect of the sulfate on the anti-complement activity was influenced by the concentrations of the samples.

\subsection{The Effect of the Content of Uronic acid (UA) on the Anti-Complement Activity}

From the above results, it was concluded that the anti-complement activities of sulfated galactofucan were better than the heteropolysaccharides with UA. To study the effect of UA content 
on the activity, three polysaccharides with different UA contents were obtained. One polysaccharide (SJW-2-R) was obtained through UA reduction, and the other two (SJW-2-HJ and SJW-2-HW) were fractionated using an activated carbon column. After reduction, the most apparent changes were in the UA content, which was decreased from $17.35 \%$ to $9.86 \%$, while the molar ratio of glucose (Glc) to Fuc was increased from 0 to 0.10 , and the glucuronic acid (GlcA) to Fuc ratio decreased from 0.18 to 0.06 . Other chemical compositions, shown in Table 1, did not change as obviously. A comparison of the activities of SJW-2 and SJW-2-R in Figure 4a indicates that the change in UA content did not influence the activity. SJW-2-HJ, which was eluted with ethanol, contained a higher UA content and lower sulfate content than that of SJW-2-HW, which was eluted with water. Moreover, SJW-2-HJ had a smaller molecular weight and a higher content of other monosaccharides. Figure 4a shows that SJW-2-HJ did not have any activity, while SJW-2-HW had better activities than that of SJW-2.

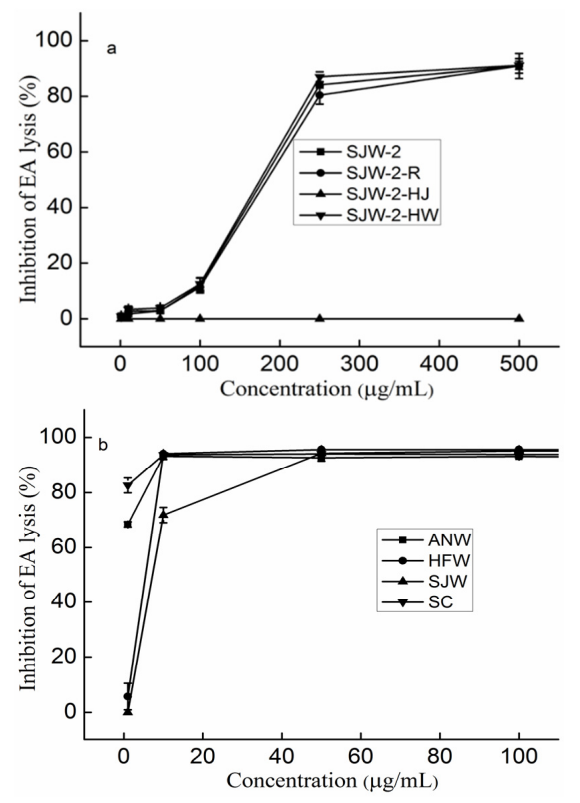

Figure 4. Inhibition of the classical pathway-mediated haemolysis of EA in 1:10-diluted NHS in the presence of increasing amounts of polysaccharides (a) Polysaccharides with different linkages; (b) Polysaccharides with branching or no branching. The results are expressed as percent inhibition of haemolysis. Data are the means from three determinations \pm S.E.M. SJW-2-R was obtained through UA reduction, and SJW-2-HJ and SJW-2-HW were fractionated using an activated carbon column. Polysaccharides derived from Ascophyllum nodosum (ANW) and Acaudina molpadioides (SC).

In summary, the UA fraction had no anti-complement activity; however, it could reduce the activity in other samples by decreasing the effective concentration of the active compositions when it was mixed with the active compositions. This might explain the observed phenomenon that the heteropolysaccharides with high UA content had lower activities.

\subsection{The Effect of the Linkage of Sulfated Galactofucan on the Anti-Complement Activity}

Crude polysaccharide (ANW) was extracted from brown algae Ascophyllum nodosum. According to previous studies [36-39], it has a backbone of alternating $\alpha(1 \rightarrow 3)$ and $\alpha(1 \rightarrow 4)$-L-Fucp residues and is sulfated at C-2 and possibly C-3 or C-4. SJW contains mainly $\alpha(1 \rightarrow 3)$-L-Fucp residues sulfated at C-4, while HFW is mainly composed of $\alpha(1 \rightarrow 3)$-L-Fucp residues sulfated at C-2 [26,27]. Figure 4b shows the anti-complement activities of ANW, SJW, and HFW. They all showed dose dependence and reached a plateau. Specifically, the $\mathrm{IC}_{50}$ values of $\mathrm{ANW}, \mathrm{SJW}$, and HFW were 0.98, 7.26, and $5.51 \mu \mathrm{g} / \mathrm{mL}$, respectively. The differences in the activities could be explained by noting that SJW had a higher UA content, while HFW had a higher content of laminaran, which had no anti-complement 
activity (as discussed in Section 3.9). Thus, it was proposed that there might be little or no effect of the linkage on the anti-complement activity.

\subsection{The Effect of the Branching of Polysaccharides on the Anti-Complement Activity}

Polysaccharide (SC) was extracted from sea cucumber Acaudina molpadioides according to a previous study [40]. It was reported [16] that polysaccharides from marine invertebrates possess a clearly linear backbone, consisting of a regular repeating unit, in contrast with the algal polysaccharides. Thus, the anti-complement activities of the polysaccharides from algae and marine invertebrates were determined to elucidate the effect of the branching. The activity of SC in Figure $4 \mathrm{~b}$ is similar to that of ANW, indicating that there was little impact of branching on the anti-complement activities. This result contrasts a previous study [41] that suggested that branching of fucoidan oligosaccharides had a major impact on their anti-complement activity. This difference might be explained based on the conformational state of the polysaccharides. The conformational states of the oligosaccharides depend on branching, while the conformational states of the polysaccharides do not. Therefore, it was concluded that there might be little or no effect of the branching of polysaccharides on the anti-complement activity.

\subsection{The Effect of the Type of Monosaccharides on the Anti-Complement Activity}

To confirm whether Fuc was necessary for the activity, sulfated laminaran (HFW-1-S) was prepared. Figure 5a shows that laminaran (both HFW-1 and HFS-1 were laminaran) had no activity, while sulfated laminaran had good activity, suggesting that Fuc was not necessary for the activity. Thus, it was concluded that laminaran, like the UA fraction, had no activity. However, it could reduce the activity by decreasing the effective concentrations of the active components. In addition, it also confirmed that the fucose residue was not necessary.
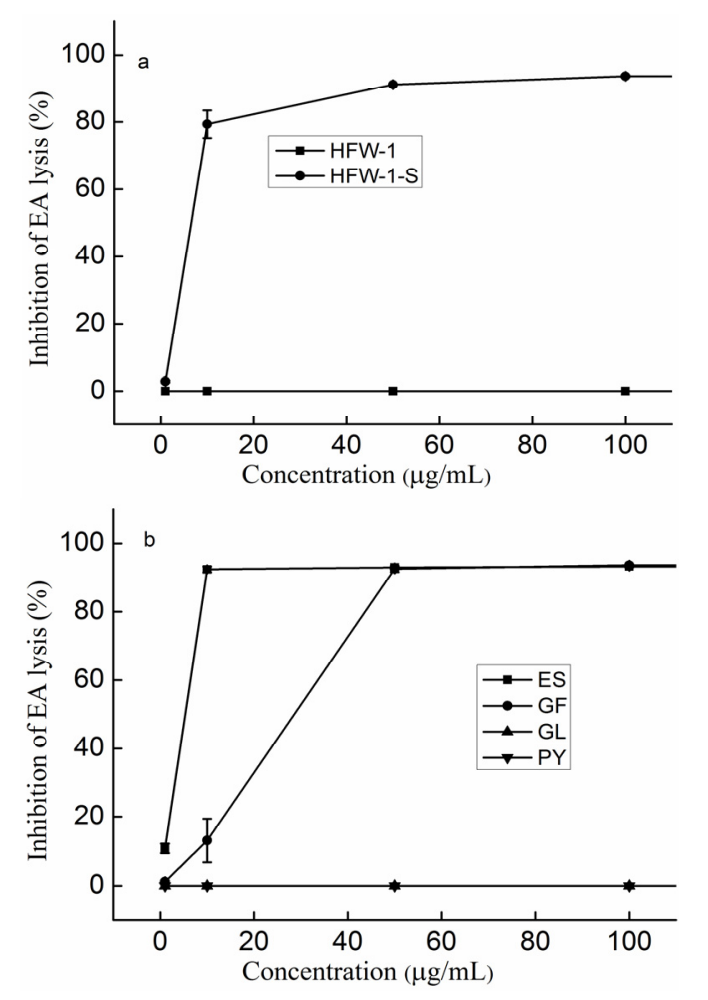

Figure 5. Cont. 


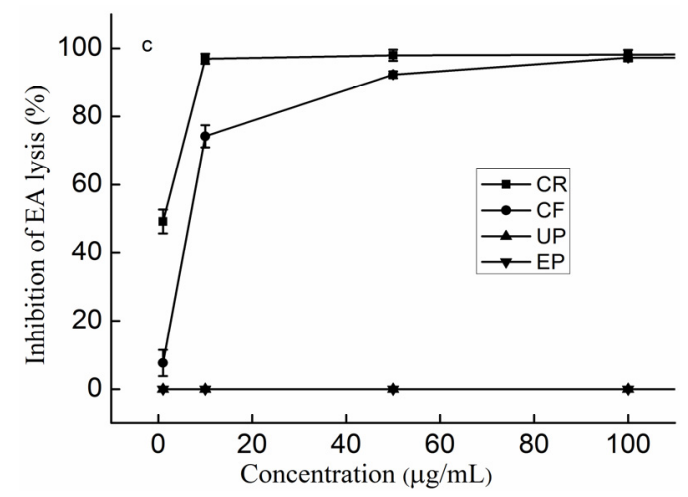

Figure 5. Inhibition of the classical pathway-mediated haemolysis of EA in 1:10-diluted NHS in the presence of increasing amounts of the polysaccharides (a) Laminaran and sulfated laminaran; (b) Polysaccharides from red algae; (c) Polysaccharides from green algae. The results are expressed as percent inhibition of haemolysis. Data are the means from three determinations \pm S.E.M. Polysaccharides derived from Eucheuma spinosum (ES), Grateloupia filicina (GF), Gracilaria lamaneiformis (GL), Porphyra yezoensis (PY), Enteromorpha prolifera (EP), Ulva pertusa (UP), Cladophera rupestris (CR) and Codium fragile (CF). SJW-2-R was obtained through UA reduction, and SJW-2-HJ and SJW-2-HW were fractionated using an activated carbon column. Overly sulfated laminaran (HFW-1-S).

Four types of crude polysaccharides from red algae, namely Grateloupia filicina (GF), Eucheuma spinosum (ES), Gracilaria lamaneiformis (GL), and Porphyra yezoensis (PY), were prepared. ES is a carrageenan polysaccharide; GF is an agar-carrageenan intermediate polysaccharide, and GL and PY are agar polysaccharides. The most apparent difference in the chemical compositions, as shown in Table 1, is the sulfate content. Figure 5b shows that ES had the best anti-complement activity, GF second, and GL and PY had no activities, indicating that sulfate was essential for the anti-complement activity. It was also confirmed that Fuc was not necessary.

Four types of crude polysaccharides from green algae, namely Cladophera rupestris (CR), Codium fragile (CF), Enteromorpha prolifera (EP), and Ulva pertusa (UP), were prepared. The analysis of the chemical compositions in Table 1 indicates that the former two belonged to type 1, while the latter two were type 2. Figure $5 \mathrm{c}$ shows that $\mathrm{CR}$ and $\mathrm{CF}$ showed good anti-complement activities, while EP and UP did not. This might be explained by the high content of UA. In addition, the solution of the polysaccharides with high UA content was too thick.

The main polysaccharides from brown algae (Saccharina japonica and Hizikia fusiforme) were laminaran, alginate, and fucoidan. Fucoidan might be classified as a combination of sulfated heteropolysaccharides and sulfated galactofucan. In the above results, it was concluded that sulfated galactofucan was the active component of the anti-complement activity. In addition, the results also showed that laminaran did not have any activity. Moreover, it was indicated that heteropolysaccharides with UA had lower activity. Alginate (data not shown) displayed no activity, which might confirm this.

Thus, it was concluded that the types of monosaccharides (neutral monosaccharides) had no effect on the anti-complement activity.

\section{Experimental Section}

\subsection{Materials}

The brown algae Saccharina japonica was collected in Rongcheng, Qingdao, China, on 28 May 2014. The brown algae Hizikia fusiforme was purchased from Dong Tou, Zhejiang, China, on 18 July 2014. The green algae Enteromorpha prolifera was collected in Qingdao, China, on 15 June 2012. The green algae Codium fragile and Ulva pertusa were collected in Qingdao, China, on 12 December 2013. The green algae Cladophera rupestris was collected in Rongcheng, Qingdao, China, 
on 24 July 2014. The red algae Porphyra yezoensis was purchased from Lianyungang, Jiangsu, China. The red algae Grateloupia filicina, Eucheuma spinosum, and Gracilaria lamaneiformis were purchased from Zhanjiang, China. Ascophyllum nodosum and Acaudina molpadioides were purchased from Qingdao, China. These were authenticated by Professor Zuhong Xu.

\subsection{Extraction and Preparation of Polysaccharides}

Brown algae (Saccharina japonica, Hizikia fusiforme, and Ascophyllum nodosum) (100 g) were cut into pieces and treated with $85 \%$ ethanol three times to remove the pigment. Crude polysaccharide was extracted from the residual material with hot water $(3 \mathrm{~L})$ for $4 \mathrm{~h}$. The extract solution was filtered with Celite and concentrated. Further elimination of algin was achieved using $20 \%$ ethanol with $\mathrm{MgCl}_{2}$ $(0.05 \mathrm{M} / \mathrm{L})$. After removing the algin, the supernatant fluid was ultra-filtered. Finally, the dialysate was concentrated, and the crude polysaccharides were obtained by ethanol precipitation and named SJW, HFW, and ANW, respectively.

Green algae and red algae (100 g) were cut into pieces and treated with $85 \%$ ethanol three times to remove the pigment. Crude polysaccharides were extracted from the residual material with hot water (3 L) for $4 \mathrm{~h}$. The extract solution was filtered with Celite, concentrated, and dialyzed. Finally, the dialysate was concentrated, and the crude polysaccharides were obtained by ethanol precipitation.

Crude polysaccharides from brown algae (Saccharina japonica and Hizikia fusiforme) were extracted from the residual material with $0.1 \mathrm{M} \mathrm{HCl}(2 \mathrm{~L})$ at room temperature for $3 \mathrm{~h}$. The extract solution was filtered with Celite, ultra-filtered, and concentrated. Finally, the crude polysaccharides were obtained by ethanol precipitation and named SJS and HFS.

Crude polysaccharide (SC) was extracted from the sea cucumber Acaudina molpadioides as previously described [40]. Briefly, crude polysaccharide was extracted from the fresh body wall $(10 \mathrm{~g})$ with hot water $(500 \mathrm{~mL})$ for $4 \mathrm{~h}$. The extract solution was filtered with Celite and concentrated. The concentrated solution was hydrolysed with papain. Papain was deactivated by hot water. Then, the solution was centrifuged, concentrated, and dialyzed. Finally, the dialysate was concentrated, and the crude polysaccharide was obtained by ethanol precipitation and named SC.

\subsection{Preparation and Purification of Polysaccharides}

Crude polysaccharides (SJW and SJS) (8 g) underwent anion exchange chromatography on a DEAE-Bio Gel Agarose FF gel $(6 \mathrm{~cm} \times 40 \mathrm{~cm})$ with elution by $0.5 \mathrm{M}(5 \mathrm{~L})(\mathrm{SJW}-1$ and SJS-1), $1 \mathrm{M}(5 \mathrm{~L})$ (SJW-2 and SJS-2), and $2 \mathrm{M} \mathrm{NaCl}$ (5 L) (SJW-3 and SJS-3). The polysaccharides were then ultrafiltrated, concentrated, and precipitated by ethanol.

Crude polysaccharides (HFW and HFS) (8 g) also underwent anion exchange chromatography on a DEAE-Bio Gel Agarose FF gel $(6 \mathrm{~cm} \times 40 \mathrm{~cm})$ with elution by water $(5 \mathrm{~L})(\mathrm{HFW}-1$ and HFS-1), $0.5 \mathrm{M}$ (5 L) (HFW-2 and HFS-2), and $2 \mathrm{M} \mathrm{NaCl}$ (5 L) (HFW-3 and HFS-3). The polysaccharides were then ultrafiltrated, concentrated, and precipitated by ethanol.

Fraction SJW-2 was fractionated using an activated carbon column $(2.6 \mathrm{~cm} \times 30 \mathrm{~cm})$ with elution by water (1 L) (SJW-2-HW) and a gradient elution from 50\% ethanol to $95 \%$ ethanol (SJW-2-HJ). The polysaccharides were concentrated and precipitated by ethanol.

\subsection{Preparation of Desulfated Polysaccharides}

The desulfated polysaccharides were prepared according to a method reported in the literature [27]. Briefly, SJS (1 g) was dissolved in distilled water $(100 \mathrm{~mL})$ and mixed with cationic resin $\left(\mathrm{H}^{+}\right)$for $3 \mathrm{~h}$. After filtration, the solution was neutralized with pyridinium and lyophilized. The solution was dissolved in dimethyl sulfoxide (DMSO): methanol (9:1; $v / v, 20 \mathrm{~mL})$. The mixture was heated at $80^{\circ} \mathrm{C}$ for $5 \mathrm{~h}$, and the desulfated products (SJS-DS) were dialyzed and lyophilized. 


\subsection{Preparation of over-Sulfated Polysaccharides}

The over-sulfated polysaccharides were prepared according to a method reported in the literature [42]. Briefly, polysaccharides (SJS, SJS-DS and HFW-1) $(1 \mathrm{~g})$ in dimethyl formamide (DMF) $(20 \mathrm{~mL})$ were treated with a sulphur trioxide pyridine complex $(5 \mathrm{~g})$ at $50{ }^{\circ} \mathrm{C}$ for $24 \mathrm{~h}$, and then the over-sulfated products (SJS-OS, SJS-DS-OS and HFW-1-S) were neutralized, dialyzed, and lyophilized.

\subsection{Preparation of the Polysaccharides with Carboxyl Reduction}

Carboxyl reduction was performed as previously described [43]. Briefly, SJW-2 (1 g) was dissolved in distilled water $(50 \mathrm{~mL})$, followed by the addition of 1-ethyl-3-(3-dimethyl-aminopropyl) carbodiimide (EDC). The solution was stirred at room temperature for $1 \mathrm{~h}$ under constant $\mathrm{pH}$ conditions (approximately 4.8) by the addition of $0.1 \mathrm{M} \mathrm{HCl}$. Then, freshly prepared $2 \mathrm{M}$ sodium borohydride $(10 \mathrm{~mL})$ was added twice during the next $2 \mathrm{~h}$ at $50^{\circ} \mathrm{C}$. Later, the reaction was terminated by the addition of glacial acetic acid. Finally, the polysaccharide with carboxyl reduction (SJW-2-R) was dialyzed, concentrated, and lyophilized.

\subsection{Compositional Analysis}

The sulfated contents were performed by ion chromatography on a Shodex IC SI- $524 \mathrm{E}$ column $(4.0 \times 250 \mathrm{~mm})$ and eluted with $3.6 \mathrm{mM} \mathrm{Na}_{2} \mathrm{CO}_{3}$ at a flow rate of $0.8 \mathrm{~mL} / \mathrm{min}$ at $45^{\circ} \mathrm{C}$. The molar ratios of monosaccharides and fucose content were determined as described by Zhang et al. [44]. The uronic acid (UA) concentration was determined by a modified carbazole method [45]. The molecular weights of the polysaccharides were evaluated by GPC-HPLC on a TSK G3000 PWxl column $(7 \mu \mathrm{m} 7.8 \times 300 \mathrm{~mm})$ with elution in $0.05 \mathrm{M} \mathrm{Na}_{2} \mathrm{SO}_{4}$ at a flow rate of $0.5 \mathrm{~mL} / \mathrm{min}$ at $40{ }^{\circ} \mathrm{C}$ with refractive index detection. Ten different molecular weight dextrans purchased from the National Institute for the Control of Pharmaceutical and Biological Products (Beijing, China) were used as weight standards.

\subsection{Anti-Complement Activity}

According to previous studies [4,46], the anti-complement activities of the polysaccharides were determined following the classical pathway. In the classical pathway, various dilutions $(100 \mu \mathrm{L})$ of the polysaccharides were mixed with 1:10 diluted normal human serum (NHS, which was obtained from healthy adult donors) $(100 \mu \mathrm{L}), \mathrm{GVB}^{2+}$ (veronal buffer saline [VBS] containing $0.1 \%$ gelatin, $0.5 \mathrm{mM} \mathrm{Mg}^{2+}$ and $\left.0.15 \mathrm{mM} \mathrm{Ca}^{2+}\right)(200 \mu \mathrm{L})$ and sensitized erythrocytes (EA) $(200 \mu \mathrm{L})$. Then, the mixture was incubated at $37{ }^{\circ} \mathrm{C}$ for $30 \mathrm{~min}$. The following assay controls were incubated under the same conditions: (1) $100 \%$ lysis: EA $(200 \mu \mathrm{L})$ in water $(400 \mu \mathrm{L})$; (2) sample control: sample $(100 \mu \mathrm{L})$ in $\mathrm{GVB}^{2+}(500 \mu \mathrm{L})$; (3) complement: 1:10-diluted NHS $(100 \mu \mathrm{L})$ and EA $(200 \mu \mathrm{L})$ in GVB ${ }^{2+}$ $(300 \mu \mathrm{L})$; and (4) blank: EA $(200 \mu \mathrm{L})$ in $\mathrm{GVB}^{2+}(400 \mu \mathrm{L})$. After incubation, the mixture was centrifuged (5000 rpm $\times 10 \mathrm{~min}$ ) and the erythrocyte lysis was determined at $405 \mathrm{~nm}$. Decreased lysis in the presence of tested polysaccharides indicated anti-complement activity. All of the samples were dissolved in $\mathrm{GVB}^{2+}$. The percent inhibition was calculated using the following equation: inhibition of EA lysis $(\%)=\left(\mathrm{A}_{\text {complement }}-\left(\mathrm{A}_{\text {sample }}-\mathrm{A}_{\text {sample control }}\right)\right) / \mathrm{A}_{\text {complement }} \times 100$.

\subsection{Statistical Analysis}

All data are shown as the mean \pm standard deviation (SD). Significant differences between the experimental groups were determined by one-way ANOVA, and differences were considered to be statistically significant if $p<0.05$. All calculations were performed using SPSS 16.0 statistical software. 


\section{Conclusions}

In summary, four crude polysaccharides (SJW, SJS, HFS, and HFW) were extracted by water and $0.1 \mathrm{M} \mathrm{HCl}$ from Saccharina japonica and Hizikia fusiforme to determine the effects of the extraction methods. The results could not definitively elucidate the effect of the extraction method. Crude polysaccharides were fractionated using anion exchange chromatography. It was found that sulfated galactofucan maintained its activity, indicating that it was the active composition. To study the effect of molecular weight, several different molecular weight polysaccharides were obtained. It was shown that the larger the molecular weight, the better the activity. However, the activity reached a plateau. In addition, the effect of the molar ratio of galactose to fucose was also considered. It was observed that the molar ratio of galactose to fucose was a positive factor. Moreover, the activities of SJS-OS, SJS-DS, and SJS-DS-OS confirmed that sulfate was an important positive factor. The activities of HFS-3 and SJS-3 suggested that the sulfation pattern had no effect at a concentration of more than $10 \mu \mathrm{g} / \mathrm{mL}$, like linkage and branching. The type of monosaccharides had no effect. Finally, it was concluded that the UA fraction and laminaran had no effect on the anti-complement activity; however, it could reduce the activity by decreasing the effective concentration of the active composition.

Acknowledgments: This study was supported by the Shandong Provincial Natural Science Foundation, China (ZR2014DQ024), National Natural Science Foundation of China (41506165), Shandong Province Science \& Technology Development Project (2014GHY115017), Qingdao Shinan District S\&T Development Fund (2013-12-009-SW), Regional Demonstration Project of Marine Economic Development in 2014, the Ocean Public Welfare Scientific Research Project, the State Oceanic Administration of the People's Republic of China (No. 201405040).

Author Contributions: Weihua Jin and Wenjing Zhang conceived and designed the experiments; Weihua Jin and Wenjing Zhang performed the experiments; Weihua Jin, Quanbin Zhang and Hongze Liang contributed reagents, materials and financial support. Weihua Jin wrote the paper. All authors have read and approved the final manuscript.

Conflicts of Interest: The authors declare no conflict of interest.

\section{References}

1. Sahu, A.; Lambris, J.D. Complement inhibitors: A resurgent concept in anti-inflammatory therapertics. Immunopharmacology 2000, 49, 133-148. [CrossRef]

2. Morgan, B.P.; Harris, C.L. Complement therapertics: History and current progress. Mol. Immunol. 2003, 40, 159-170. [CrossRef]

3. Maillet, F.; Maurice, P.; Jean, C.; Kazatchkine, M.D. Structure-function relationships in the inhibitory effect of heparin on complement activation: Independency of the anti-coagulant and anti-complementary sites on the heparin molecule. Mol. Immunol. 1988, 25, 917-923. [CrossRef]

4. Xu, H.; Zhang, Y.; Zhang, J.; Chen, D. Isolation and characterization of an anti-complementary polysaccharide D3-S1 from the roots of Bupleurum smithii. Int. Immunopharmacol. 2007, 7, 175-182. [CrossRef] [PubMed]

5. Fang, X.B.; Yin, X.X.; Yuan, G.F.; Chen, X.O. Chemical and biological characterization of polysaccharides from the bark of Avicennia marina. Eur. Food Res. Technol. 2015, 241, 17-25. [CrossRef]

6. Samuelsena, A.B.; Lunda, I.; Djahromia, J.M.; Paulsena, B.S.; Wolda, J.K.; Knutsen, H.S. Structural features and anti-complementary activity of some heteroxylan polysaccharide fractions from the seeds of Plantago major L. Carbohydr. Polym. 1999, 38, 133-143. [CrossRef]

7. Ni, F.; Liu, L.; Song, Y.; Wang, X.; Zhao, Y.; Huang, W.; Wang, Z.; Xiao, W. Anti-complementary phenolic acids from Lonicera japonica. China J. Chin. Mater. Med. 2015, 40, 269-274.

8. Sun, Q.; Bao, J. Purification, cloning and characterization of a metalloproteinase from Naja atra venom. Toxicon 2015, 56, 1459-1469. [CrossRef] [PubMed]

9. Makrides, S.C. Therapeutic Inhibition of the Complement System. Pharmacological 1998, 50, 59-88.

10. Xu, X.; Chen, L.; Zhao, X. Review on the research and development of anti-complementary agents from natural products. Nat. Prod. Res. Dev. 2015, 27, 355.

11. Mauzac, M.; Maillet, F.; Jozefonvicz, J.; Kazatchkine, M.D. Anticomplementary activity of dextran derivatives. Biomaterials 1985, 6, 61-63. [CrossRef] 
12. Crepon, B.; Maillet, F.; Kazatchkine, M.D.; Jozefonvicz, J. Molecular weight dependency of the acquired anticomplementary and anticoagulant activities of specifically substituted dextrans. Biomaterials 1987, 8 , 248-253. [CrossRef]

13. Blondin, C.; Chaubet, F.; Nardella, A.; Sinquin, C.; Jozefonvicz, J. Relationships between chemical characteristics and anticomplementary activity of fucans. Biomaterials 1996, 17, 597-603. [CrossRef]

14. Zhang, W.; Jin, W.; Sun, D.; Zhao, L.; Wang, J.; Duan, D.; Zhang, Q. Structural analysis and anti-complement activity of polysaccharides from Kjellmaniella crsaaifolia. Mar. Drugs 2015, 13, 1360-1374. [CrossRef] [PubMed]

15. Blondin, C.; Fisher, E.; Boisson-Vadal, C.; Kazatchkine, M.D.; Jozefonvicz, J. Inhibition of complement activation by natural sulfated polysaccharides (fucans) from brown seaweed. Mol. Immunol. 1994, 31, 247-253. [CrossRef]

16. Berteau, O.; Mulloy, B. Sulfated fucans, fresh perspectives: Structures, functions, and biological properties of sulfated fucans and an overview of enzymes active toward this class of polysaccharide. Glycobiology 2003, 13, 29R-40R. [CrossRef] [PubMed]

17. Bilan, M.I.; Grachev, A.A.; Shashkov, A.S.; Kelly, M.; Sanderson, C.J.; Nifantiev, N.E.; Usov, A.I. Further studies on the composition and structure of a fucoidan preparation from the brown alga Saccharina latissima. Carbohydr. Res. 2010, 345, 2038-2047. [CrossRef] [PubMed]

18. Jiao, G.; Yu, G.; Zhang, J.; Ewart, H. Chemical structures and bioactivities of sulfated polysaccharides from marine algae. Mar. Drugs 2011, 9, 196-223. [CrossRef] [PubMed]

19. Kusaykin, M.; Bakunina, I.; Sova, V.; Ermakova, S.; Kuznetsova, T.; Besednova, N.; Zaporozhets, T.; Zvyagintseva, T. Structure, biological activity, and enzymatic transformation of fucoidans from the brown seaweeds. Biotechnol. J. 2008, 3, 904-915. [CrossRef] [PubMed]

20. Li, B.; Lu, F.; Wei, X.; Zhao, R. Fucoidan: Structure and Bioactivity. Molecules 2008, 13, 1671-1695. [CrossRef] [PubMed]

21. Pomin, V.H.; Mourao, P.A.S. Structure, biology, evolution, and medical importance of sulfated fucans and galactans. Glycobiology 2008, 18, 1016-1027. [CrossRef] [PubMed]

22. Lahaye, M. Developments on gelling algal galactans, their structure and physico-chemistry. J. Appl. Phycol. 2001, 13, 173-184. [CrossRef]

23. Pomin, V.H. Structural and functional insights into sulfated galactans: A systematic review. Glycoconj. J. 2010, 27, 1-12. [CrossRef] [PubMed]

24. USov, A.I. Structural analysis of red seaweed galactans of agar and carrageenan groups. Food Hydrocoll. 1998, 12, 301-308. [CrossRef]

25. Lahaye, M.; Robic, A. Structure and Functional Properties of Ulvan, a Polysaccharide from Green Seaweeds. Biomacromolecules 2007, 8, 1765-1774. [CrossRef] [PubMed]

26. Jin, W.; Zhang, W.; Wang, J.; Ren, S.; Song, N.; Duan, D.; Zhang, Q. Characterization of laminaran and a highly sulfated polysaccharide from Sargassum fusiforme. Carbohydr. Res. 2014, 385, 58-64. [CrossRef] [PubMed]

27. Jin, W.; Zhang, W.; Wang, J.; Ren, S.; Song, N.; Zhang, Q. Structural analysis of heteropolysaccharide from Saccharina japonica and its derived oligosaccharides. Int. J. Biol. Macromol. 2013, 62, 697-704. [CrossRef] [PubMed]

28. Jin, W.; Guo, Z.; Wang, J.; Zhang, W.; Zhang, Q. Structural analysis of sulfated fucan from Saccharina japonica by electrospray ionization tandem mass spectrometry. Carbohydr. Res. 2013, 369, 63-67. [CrossRef] [PubMed]

29. Jin, W.; Wang, J.; Ren, S.; Song, N.; Zhang, Q. Structural analysis of a heteropolysaccharide from Saccharina japonica by electrospray mass spectrometry in tandem with collision-induced dissociation tandem mass spectrometry (ESI-CID-MS/MS). Mar. Drugs 2012, 10, 2138-2152. [CrossRef] [PubMed]

30. Jin, W.; Zhang, Q.; Wang, J.; Zhang, W. A comparative study of the anticoagulant activities of eleven fucoidans. Carbohydr. Polym. 2013, 91, 1-6. [CrossRef] [PubMed]

31. Cumashi, A.; Ushakova, N.A.; Preobrazhenskaya, M.E.; D’Incecco, A.; Piccoli, A.; Totani, L.; Tinari, N.; Morozevich, G.E.; Berman, A.E.; Bilan, M.I.; et al. A comparative study of the anti-inflammatory, anticoagulant, antiangiogenic, and antiadhesive activities of nine different fucoidans from brown seaweeds. Glycobiology 2007, 17, 541-552. [CrossRef] [PubMed] 
32. Karmakar, P.; Pujol, C.A.; Damonte, E.B.; Ghosh, T.; Ray, B. Polysaccharides from Padina tetrastromatica: Structural features, chemical modification and antiviral activity. Carbohydr. Polym. 2010, 80, 513-520. [CrossRef]

33. Pereira, M.S.; Melo, F.R.; Mourão, P.A.S. Is there a correlation between structure and anticoagulant action of sulfated galactans and sulfated fucans? Glycobiology 2002, 12, 573-580. [CrossRef] [PubMed]

34. Li, B.; Xue, X.J.; Sun, J.L.; Xu, S.Y. Structural investigation of a fucoidan containing a fucose-free core from the brown seaweed Hizikia fusiforme. Carbohydr. Res. 2006, 341, 1135-1146. [CrossRef] [PubMed]

35. Wang, P.; Zhao, X.; Lv, Y.; Liu, Y.; Lang, Y.; Wu, J.; Liu, X.; Li, M.; Yu, G. Analysis of structural heterogeneity of fucoidan from Hizikia fusiforme by ES-CID-MS/MS. Carbohydr. Polym. 2012, 90, 602-607. [CrossRef] [PubMed]

36. Chevolot, L.; Foucault, A.; Chaubet, F.; Kervarec, N.; Sinquin, C.; Fisher, A.M.; Boisson-Vidal, C. Further data on the structure of brown seaweed fucans: Relationships with anticoagulant activity. Carbohydr. Res. 1999, 319, 154-156. [CrossRef]

37. Chevolot, L.; Mulloy, B.; Ratiskol, J.; Foucault, A.; Colliec-Jouaultb, S. A disaccharide repeat unit is the major structure in fucoidans from two species of brown algae. Carbohydr. Res. 2001, 330, 529-535. [CrossRef]

38. Daniel, R.; Berteau, O.; Jozefonvicz, J.; Goasdoue, N. Degradation of algal (Ascophyllum nodosum) fucoidan by an enzymatic activity contained in digestive glands of the marine mollusc Pecten maximus. Carbohydr. Res. 1999, 322, 291-297. [CrossRef]

39. Daniel, R.; Berteau, O.; Chevolot, L.; Varenne, A.; Gareil, P.; Goasdoue, N. Regioselective desulfation of sulfated L-fucopyranoside by a new sulfoesterase from the marine mollusk Pecten maximus. Application to the structural study of algal fucoidan (Ascophyllum nodosum). Eur. J. Biochem. 2001, 268, 5617-5626. [CrossRef] [PubMed]

40. Xue, C.; Tang, Q.; Li, D.; Wu, X.; Wang, J. Isolation and characterization of a sea cucumber fucoidan-utilizing marine bacterium. Lett. Appl. Microbiol. 2010, 50, 301-307.

41. Clement, M.J.; Tissot, B.; Chevolot, L.; Adjadj, E.; Du, Y.; Curmi, P.A.; Daniel, R. NMR characterization and molecular modeling of fucoidan showing the importance of oligosaccharide branching in its anticomplementary activity. Glycobiology 2010, 20, 883-894. [CrossRef] [PubMed]

42. Nifantiev, N.; Ustyuzhanina, N.; Krylov, V.; Grachev, A.; Gerbst, A. Synthesis, NMR and conformational studies of fucoidan fragments, 8: Convergent synthesis of branched and linear oligosaccharides. Synthesis 2006, 23, 4017-4031. [CrossRef]

43. Kariya, Y.; Watabe, S.; Kyogashima, M.; Ishihara, M.; Ishii, T. Structure of fucose branches in the glycosaminoglycan from the body wall of the sea cucumber Stichopus japonicus. Carbohydr. Res. 1997, 297, 273-279. [CrossRef]

44. Zhang, J.; Zhang, Q.; Wang, J.; Shi, X.; Zhang, Z. Analysis of the monosaccharide composition of fucoidan by precolumn derivation HPLC. Chin. J. Oceanol. Limnol. 2009, 27, 1-5. [CrossRef]

45. Bitter, T.; Muir, H.M. A modified uronic acid carbazole reaction. Anal. Biochem. 1962, 4, 330-334. [CrossRef]

46. Klerx, J.P.A.M.; Beukelman, C.J.; Dijk, H.V.; Willers, J.M.N. Microassay for colorimetric estimation of complement activity in guinea pig, human and mouse serum. J. Immunol. Methods 1983, 63, 215-220. [CrossRef]

(C) 2015 by the authors; licensee MDPI, Basel, Switzerland. This article is an open access article distributed under the terms and conditions of the Creative Commons by Attribution (CC-BY) license (http:/ / creativecommons.org/licenses/by/4.0/). 